\title{
Fatty Acids Are Required for Epidermal Permeability Barrier Function
}

\author{
Man Mao-Qiang, Peter M. Elias, and Kenneth R. Feingold \\ Departments of Dermatology and Medicine, University of California, San Francisco, California 94143; and Dermatology Service and \\ Metabolism Section, Medical Service, Department of Veterans Affairs Medical Center, San Francisco, California 94121
}

\begin{abstract}
The permeability barrier is mediated by a mixture of ceramides, sterols, and free fatty acids arranged as extracellular lamellar bilayers in the stratum corneum. Whereas prior studies have shown that cholesterol and ceramides are required for normal barrier function, definitive evidence for the importance of nonessential fatty acids is not available. To determine whether epidermal fatty acid synthesis also is required for barrier homeostasis, we applied 5-(tetradecyloxy)-2-furancarboxylic acid (TOFA), an inhibitor of acetyl CoA carboxylase, after disruption of the barrier by acetone or tape stripping. TOFA inhibits epidermal fatty acid by $\sim \mathbf{5 0 \%}$ and significantly delays barrier recovery. Moreover, coadministration of palmitate with TOFA normalizes barrier recovery, indicating that the delay is due to a deficiency in bulk fatty acids. Furthermore, TOFA treatment also delays the return of lipids to the stratum corneum and results in abnormalities in the structure of lamellar bodies, the organelle which delivers lipid to the stratum corneum. In addition, the organization of secreted lamellar body material into lamellar bilayers within the stratum corneum interstices is disrupted by TOFA treatment. Finally, these abnormalities in lamellar body and stratum corneum membrane structure are corrected by coapplication of palmitate with TOFA. These results demonstrate a requirement for bulk fatty acids in barrier homeostasis. Thus, inhibiting the epidermal synthesis of any of the three key lipids that form the extracellular, lipid-enriched membranes of the stratum corneum results in an impairment in barrier homeostasis. (J. Clin. Invest. 1993. 92:791-798.) Key words: transepidermal water loss • fatty acid synthesis • 5-(tetradecyloxy)-2-furancarboxylic acid - stratum corneum lipids • permeability barrier
\end{abstract}

\section{Introduction}

The cutaneous permeability barrier is essential for terrestrial life. This permeability barrier is located in the stratum corneum and is mediated by lipid-enriched membrane multilayers with a characteristic lamellar repeat structure within the intercellular spaces (1). The lipids in the intercellular domains consist primarily of ceramides, cholesterol, and free fatty acids (1). Topical application of organic solvents, such as acetone, or tape stripping remove these lipids from the stratum corneum,

Address correspondence to Kenneth R. Feingold, M. D., Metabolism Section (111F), Department of Veterans Affairs Medical Center, 4150 Clement Street, San Francisco, CA 94121.

Received for publication 4 August 1992 and in revised form 28 January 1993.

The Journal of Clinical Investigation, Inc.

Volume 92, August 1993, 791-798 thereby acutely disrupting the permeability barrier (2). Such acute perturbations initiate a sequence of events that rapidly results in the return of lipids to the stratum corneum and the restoration of barrier function (2). These events include: $(a)$ the rapid secretion of preformed lamellar bodies by the stratum granulosum cells ( 3$) ;(b)$ an increase in cholesterol, sphingolipid, and fatty acid synthesis in the epidermis $(2,4-6)$; and $(c)$ the increased formation and accelerated secretion of newly synthesized lamellar bodies by the stratum granulosum cells (3). The increase in both cholesterol and fatty acid synthesis in the epidermis occurs within an hour or two after disruption of the barrier, whereas the increase in sphingolipid synthesis is delayed $(6 \mathrm{~h}$ or later) $(2,4-6)$.

To assess the role of specific lipids in the barrier further, we have used inhibitors of lipid synthesis. Blocking the synthesis of cholesterol with competitive inhibitors of $\mathrm{HMG} \mathrm{CoA}$ reductase, such as lovastatin or fluvastatin, delays barrier recovery after acetone treatment, which is associated with a decrease in the return of cholesterol to the stratum corneum $(7,8)$. Moreover, either mevalonate, the immediate product of the enzyme, or cholesterol, the final product of the biosynthetic pathway overcomes the inhibition and restores barrier recovery to normal indicating that the delay in barrier recovery is due to the inhibition of HMG CoA reductase (7). Likewise, inhibition of sphingolipid synthesis, with $\beta$-choloroalanine, an inhibitor of serine palmitoyl transferase, delays barrier repair (9). The decrease in barrier repair in the $\beta$-chloroalanine-treated animals is associated with a decrease in the return of sphingolipids to the stratum corneum (9). Barrier repair rates are normalized by providing exogenous sphingolipids demonstrating that the effect of $\beta$-chloroalanine on barrier repair is due to sphingolipid synthesis inhibition rather than a nonspecific toxic effect (9).

Although these studies indicate that cholesterol and sphingolipids are required for barrier homeostasis, little is known regarding the requirement for nonessential fatty acids, the third remaining major class of lipids in the stratum corneum lipid mixture. Whereas the essential fatty acid, linoleate, is required for barrier function, it largely occupies the w-esterified position on acyl ceramides $(10,11)$. Yet, as noted above, previous studies demonstrated that disruption of the barrier stimulates bulk epidermal fatty acid synthesis $(2,5)$. The purpose of the present study was to determine if inhibiting epidermal fatty acid synthesis after acute perturbations of barrier function would delay barrier repair, demonstrating a requirement not only for essential fatty acids, but also bulk free fatty acids in barrier homeostasis.

\section{Methods}

Materials. Male hairless mice $(\mathrm{hr} / \mathrm{hr}), 8-12$ wk old, were purchased from Simonsen Laboratories (Gilroy, CA) and fed mouse diet (Ralston-Purina Co., St. Louis, MO) and water ad lib. Acetone and propyl- 
ene glycol were purchased from Fisher Scientific (Fairlane, NJ). Palmitic acid methyl ester was purchased from Sigma Chemical Co. (St. Louis, MO). Ruthenium tetroxide was purchased from Polysciences Inc. (Warrington, PA). Tritiated water $(5 \mathrm{Ci} / \mathrm{ml})$ and $\left[{ }^{14} \mathrm{C}\right]$ acetate $(60$ $\mathrm{mCi} / \mathrm{mmol}$ ) were purchased from New England Nuclear (Boston, MA). Thin layer chromatography polygram Sil G plates were purchased from Brinkmann Instruments (Westbury, NY). Cytosint scintillation liquid was purchased from ICN Pharmaceuticals, Inc. (Irvine, CA). Fluvastatin (XU62-320, fluindostatin) was a gift from Dr. A. Stutz, Sandoz Research Institute, Vienna, Austria. 5-tetradecyloxy-2furancarboxylic acid (TOFA) ${ }^{1}$ (MDL 14514) was a gift from Dr. E. Bohme, Marion Merrill Dow Research Institute, Marion Merrill Dow Inc., Cincinnati, OH. Epicholesterol was purchased from Research Plus Steroid Laboratories (Denville, NJ).

Animal procedures. Male hairless mice were treated with absolute acetone to disrupt the barrier while under anesthesia as previously described (3-6). Alternatively, barrier disruption was achieved by repeated application of cellophane tape (four to six times). Both procedures were terminated when transepidermal water loss (TEWL) rates reached $2-6 \mathrm{mg} / \mathrm{cm}^{2}$ per $\mathrm{h}$, as measured with an electrolytic water analyzer (Meeco Inc., Warrington, PA). Immediately after acetone treatment or tape stripping the entire flank of cohorts of animals were treated with: $(a)$ vehicle consisting of propylene glycol:ethanol 70:30 $\mathrm{vol} / \mathrm{vol} ;(b)$ fluvastatin, $400 \mu \mathrm{g}$ in vehicle; $(c)$ TOFA, $130 \mu \mathrm{g}$ in vehicle; (d) palmitic acid, $200 \mu \mathrm{g}$ in vehicle; $(e)$ cholesterol, $400 \mu \mathrm{g}$ in vehicle; $(f)$ epicholesterol, $400 \mu \mathrm{g}$ in vehicle; or $(g)$ combinations of the above. TEWL was measured at 2,4 , and $6 \mathrm{~h}$ after disrupting the barrier and drug application. Because the extent of barrier disruption varied from animal to animal, barrier recovery is expressed as percent of maximal TEWL (value immediately after disrupting barrier) rather than in absolute values.

Lipid synthesis. The effect of TOFA and fluvastatin on fatty acid and cholesterol synthesis in vitro and in vivo was assessed in mice after disrupting the barrier with either acetone treatment or tape stripping. In the in vivo studies, tritiated water $(20 \mathrm{mCi} / 0.2 \mathrm{ml})$ was administered $1 \mathrm{~h}$ after barrier disruption. $2 \mathrm{~h}$ later, blood samples were taken and the animals killed. Whole skin was excised and the epidermis separated from the dermis by heat separation, as described previously (4, 5 ). The incorporation of tritiated water into cholesterol and fatty acids was determined after saponification, extraction with petroleum ether, and thin layer chromatography as described in detail in previous publications $(4,5)$. The specific activity of tritiated water in serum samples was used to calculate the incorporation rates. Results are presented as nanomoles product formed per 2 hours per milligram of epidermal wet weight. In the in vitro studies, the animals were killed $1 \mathrm{~h}$ after barrier disruption and skin samples were incubated for $2 \mathrm{~h}$ at $37^{\circ} \mathrm{C}$ in a $2-\mathrm{ml}$ solution of $10 \mathrm{mM}$ EDTA in Dulbecco's PBS, calcium and magnesium free, containing $40 \mu \mathrm{Ci}\left[{ }^{14} \mathrm{C}\right]$ acetate, as described previously $(12,13)$. After stopping the reaction by immersion in iced PBS, the epidermis was separated from the dermis and the quantity of labeled fatty acids and cholesterol determined in the epidermis after saponification, extraction, and thin layer chromatography, as described previously $(4,5$, 13). Results are expressed as nanomoles incorporated per hour per gram epidermis. Because of variations in the rates of lipid synthesis from month to month, only the results from animals that were studied simultaneously under identical conditions are compared.

Histochemistry. Skin biopsies were taken from mice at the indicated times after barrier disruption with acetone plus various topical treatments. Samples were snap frozen in liquid nitrogen, sectioned at 4 $\mu \mathrm{m}$, and stained with Nile red for the depiction of lipid distribution in skin (14). Sections were visualized in a microscope equipped for epifluorescence (Ortholux II; E. Leitz, Inc., Rockleigh, NJ).

1. Abbreviations used in this paper: TEWL, transepidermal water loss; TOFA, 5-(tetradecyloxy)-2-furancarboxylic acid.
Electron microscopy. Samples were taken from control and treated areas $2 \mathrm{~h}$ after various treatments, minced to $0.5 \mathrm{~cm}^{2}$, fixed in modified Karnovsky's fixative overnight, washed in $0.1 \mathrm{M}$ cacodylate buffer and postfixed in $0.2 \%$ ruthenium tetroxide (Ru04) containing 0.5 potassium ferrocyanide in $0.1 \mathrm{M}$ cacodylate buffer for $30 \mathrm{~min}$ as recently described (15). 600-800-nm ultrathin sections were examined in an electron microscope (10A; Carl Zeiss Inc., Thornwood, NY) operating at $60 \mathrm{KV}$.

Statistical significance was determined using the Student's $t$ test. A two-tailed $t$ test was used when the direction of change was unknown. When the direction of change was known from previous experiments a one-tailed $t$ test was used.

\section{Results}

Effects of TOFA and fluvastatin on lipid synthesis. To determine whether topical TOFA treatment inhibits epidermal fatty acid synthesis, we measured $\left[{ }^{14} \mathrm{C}\right]$ acetate incorporation into epidermal lipids in vitro (Table I). As reported previously (2, 4,5 ), disruption of the barrier by acetone treatment stimulates both fatty acid and cholesterol synthesis in the epidermis ( $\mathrm{Ta}$ ble I, line 1 vs. line 2). Similarly, disruption of the barrier by tape stripping also increases epidermal fatty acid and cholesterol synthesis (Table I, line 4 vs. line 5). With TOFA treatment, after acetone disruption of the barrier, the incorporation of $\left[{ }^{14} \mathrm{C}\right]$ acetate into fatty acids is decreased $44 \%$, while $\left[{ }^{14} \mathrm{C}\right]-$

Table I. Lipid Synthesis In Vitro

\begin{tabular}{|c|c|c|}
\hline & Fatty acids & Cholesterol \\
\hline & \multicolumn{2}{|c|}{$\begin{array}{c}\text { nmol incorporated/h } \\
\text { per } \mathrm{g} \text { epidermis }\end{array}$} \\
\hline \multicolumn{3}{|l|}{ Experiment 1} \\
\hline 1) Untreated + vehicle $(n=6)$ & $19.1 \pm 1.58$ & $8.54 \pm 0.31$ \\
\hline 2) Acetone + vehicle $(n=6)$ & $31.7 \pm 4.56$ & $15.3 \pm 2.83$ \\
\hline 3) Acetone + TOFA $(n=6)$ & $17.8 \pm 1.58$ & $12.2 \pm 2.14$ \\
\hline 1 vs. 2 & $P<0.05$ & $P<0.05$ \\
\hline 2 vs. 3 & $P<0.02$ & NS \\
\hline \multicolumn{3}{|l|}{ Experiment 2} \\
\hline 4) Untreated + vehicle $(n=3)$ & $9.29 \pm 1.08$ & $6.50 \pm 1.10$ \\
\hline 5) Tape stripping + vehicle $(n=3)$ & $21.6 \pm 1.65$ & $15.2 \pm 1.16$ \\
\hline 6) Tape stripping + TOFA $(n=3)$ & $6.53 \pm 0.52$ & $11.2 \pm 1.17$ \\
\hline 4 vs. 5 & $P<0.01$ & $P<0.01$ \\
\hline 5 vs. 6 & $P<0.001$ & NS \\
\hline \multicolumn{3}{|l|}{ Experiment 3} \\
\hline 7) Acetone + vehicle $(n=3)$ & $16.0 \pm 4.52$ & $4.52 \pm 1.70$ \\
\hline 8) Acetone + fluvastatin $(n=3)$ & $10.4 \pm 1.84$ & $0.72 \pm 0.17$ \\
\hline 7 vs. 8 & NS & $P<0.05$ \\
\hline \multicolumn{3}{|l|}{ Experiment 4} \\
\hline 9) Tape stripping + vehicle $(n=3)$ & $8.24 \pm 2.50$ & $1.19 \pm 0.22$ \\
\hline 10) Tape stripping + fluvastatin $(n=3)$ & $10.9 \pm 2.21$ & $0.60 \pm 0.14$ \\
\hline 9 vs. 10 & NS & $P<0.05$ \\
\hline
\end{tabular}

The cutaneous permeability barrier was disrupted by either acetone treatment or tape stripping followed by the indicated treatment. $1 \mathrm{~h}$ after barrier disruption, the animals were killed and skin samples incubated in $\left[{ }^{14} \mathrm{C}\right]$ acetate for $2 \mathrm{~h}$ at $37^{\circ} \mathrm{C}$. The epidermis was separated from the dermis and the incorporation of acetate into fatty acids and cholesterol in the epidermis determined after saponification, extraction, and thin layer chromatography. $n=$ number of animals. 
acetate incorporation into cholesterol is not significantly altered (Table I, line 2 vs. line 3). Moreover, after acutely disrupting the barrier by an alternative physical method, cellophane tape stripping, topical TOFA treatment also inhibits fatty acid synthesis ( $70 \%$ decrease ) while not significantly altering the incorporation of $\left[{ }^{14} \mathrm{C}\right]$ acetate into cholesterol (Table I, line 5 vs. line 6 ). Thus, TOFA treatment abolishes the increase in epidermal fatty acid synthesis induced by disrupting the barrier by either acetone treatment or tape stripping.

After barrier disruption by either acetone treatment or tape stripping, fluvastatin inhibits cholesterol synthesis without significantly altering fatty acid synthesis ( Table I, line 7 vs. line 8 , line 9 vs. line 10). This result is similar to that previously observed after topical lovastatin administration (7).

Previous studies have demonstrated that after acetone disruption of the barrier, the in vivo incorporation of ${ }^{3} \mathrm{H}_{2} \mathrm{O}$ into cholesterol and fatty acids is increased approximately two- to threefold in the epidermis $(4,5)$. Similarly, disruption of the barrier by tape stripping also stimulates the incorporation of ${ }^{3} \mathrm{H}_{2} \mathrm{O}$ into cholesterol and fatty acids in the epidermis (cholesterol: control $0.107 \pm 0.041$ vs. tape stripped $1.24 \pm 0.381 P$ $<0.01$; fatty acids: control $8.00 \pm 1.27$ vs. tape stripped $12.24 \pm 2.12 \mathrm{nmol}$ incorporated $/ 2 \mathrm{~h}$ per mg epidermis $P<.05$, $n=9$ for each group).

To further confirm that topical TOFA inhibits fatty acid synthesis in the epidermis after barrier disruption, we also determined the effect of this compound on ${ }^{3} \mathrm{H}_{2} \mathrm{O}$ incorporation

Table II. Lipid Synthesis In Vivo

\begin{tabular}{lcc}
\hline & Fatty acids & Cholesterol \\
\hline & $n$ nol incorporated/2 & h per mg epidermis \\
Acetone treatment & & \\
Experiment 1 & & \\
1) Vehicle $(n=3)$ & $10.32 \pm 1.93$ & $0.79 \pm 0.22$ \\
2) TOFA $(n=3)$ & $4.74 \pm 0.76$ & $0.49 \pm 0.09$ \\
& $P<0.05$ & $\mathrm{NS}$ \\
Experiment 2 & & \\
3) Vehicle $(n=6)$ & $11.2 \pm 1.63$ & $0.573 \pm 0.194$ \\
4) Fluvastatin $(n=6)$ & $10.9 \pm 2.01$ & $0.160 \pm 0.058$ \\
& $\mathrm{NS}$ & $P<0.05$ \\
Tape stripping & & \\
Experiment 1 & & $1.24 \pm 0.21$ \\
5) Vehicle $(n=3)$ & $19.03 \pm 1.22$ & $0.88 \pm 0.03$ \\
6) TOFA $(n=3)$ & $8.33 \pm 0.72$ & $\mathrm{NS}$ \\
& $P<0.001$ & \\
Experiment 2 & & $0.284 \pm 0.077$ \\
7) Vehicle $(n=3)$ & $19.2 \pm 1.5$ & $0.068 \pm 0.012$ \\
8) Fluvastatin $(n=3)$ & $15.8 \pm 1.0$ & \\
& $\mathrm{NS}$ &
\end{tabular}

The cutaneous permeability barrier was disrupted by either acetone treatment or tape stripping followed by the indicated treatment. $1 \mathrm{~h}$ after barrier disruption, the animals were injected with $20 \mathrm{mCi}^{3} \mathrm{H}_{2} \mathrm{O}$. $2 \mathrm{~h}$ later, the animals were killed, the skin excised, and the epidermis separated from the dermis by heat separation. The incorporation of ${ }^{3} \mathrm{H}_{2} \mathrm{O}$ in cholesterol and fatty acids was determined after saponification, extraction, and thin layer chromatography. $n=$ number of animals. into lipids in vivo (Table II). With TOFA treatment, after acetone disruption of the barrier, the incorporation of tritiated water into fatty acids in the epidermis of intact mice is decreased $55 \%$ in comparison to animals treated with vehicle (line 1 vs. line 2). Epidermal cholesterol synthesis also appears to be decreased in the TOFA-treated animals compared to controls, but this difference does not achieve statistical significance. After disruption of the barrier by tape stripping, TOFA also significantly inhibits the incorporation of tritiated water into fatty acids in the epidermis ( $56 \%$ decrease) while not significantly affecting cholesterol synthesis (Table II, line 5 vs. line 6). Furthermore, topical fluvastatin inhibits cholesterol synthesis in the epidermis without altering fatty acid synthesis after disruption of the barrier by acetone treatment or tape stripping (Table II, line 3 vs. line 4 , line 7 vs. line 8 ). Finally, topical TOFA treatment of animals with normal barrier function does not alter tritiated water incorporation into fatty acids in the epidermis (Vehicle $(n=3): 12.9 \pm 3.5$ vs. TOFA ( $n$ $=3$ ): $15.6 \pm 1.9 \mathrm{nmol}^{3} \mathrm{H}_{2} \mathrm{O}$ incorporated $/ 2 \mathrm{~h}$ per mg epidermis; NS). Thus, in animals with a perturbed barrier, TOFA causes a marked inhibition of epidermal fatty acid synthesis, but exhibits only modest effects on cholesterol synthesis. In contrast, TOFA has no effect on epidermal lipid synthesis in animals with normal barrier function.

Effects of TOFA on permeability barrier recovery. Having shown that TOFA treatment inhibits fatty acid synthesis after barrier perturbation, we next determined whether such inhibition would lead to alterations in barrier recovery. After barrier disruption with acetone treatment, transepidermal water loss rapidly recovers towards normal, such that by $6 \mathrm{~h}$ TEWL levels normalize by $40-50 \%$ (Table III). This rapid recovery of barrier function is similar to results previously reported by this laboratory $(7-9,14)$. In contrast, a single application of topical TOFA significantly impairs barrier recovery at 2,4 , and $6 \mathrm{~h}$ (Table III).

To determine whether the effect of TOFA is due to inhibition of fatty acid synthesis or instead attributable to toxicity or unrelated effects of the drug, we next applied palmitic acid simultaneously with TOFA. As seen in Table III, barrier recov-

Table III. Effect of TOFA on Barrier Recovery

\begin{tabular}{ccc}
$2 \mathrm{~h}$ & $4 \mathrm{~h}$ & $6 \mathrm{~h}$ \\
\hline \multicolumn{4}{c}{ percent maximal TEWL }
\end{tabular}

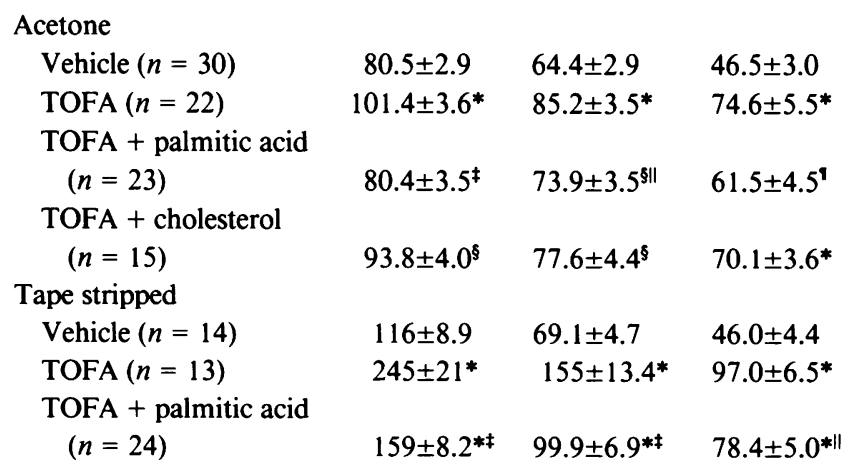

${ }^{*} P<0.001$ vs. vehicle. ${ }^{\ddagger} P<0.001$ vs. TOFA. ${ }^{\S} P<0.05$ vs. vehicle. ${ }^{\|} P<0.05$ vs. TOFA. ' $P<0.01$ vs. vehicle. 
ery improves when palmitic acid is coapplied with TOFA to acetone-treated animals. In contrast, simultaneous applications of cholesterol with TOFA does not significantly improve barrier recovery. These results indicate first that the defect in barrier recovery induced by TOFA is not due to unrelated drug effects or toxicity, and second, that the delay is primarily due to inhibition of fatty acid synthesis, since TOFA effects can be overcome by providing exogenous fatty acids alone.

To determine whether the inhibition in barrier recovery produced by TOFA treatment is related to barrier recovery in general or other responses to acetone treatment, we next determined the effect of topical TOFA treatment in an unrelated acute model of barrier disruption, cellophane tape stripping. As shown in Table III, TOFA treatment delays barrier recovery after tape stripping when compared with animals treated with vehicle alone. Moreover, coadministration of palmitic acid with TOFA also partially restores barrier recovery in this model, as in the acetone-treated animals. These results provide further evidence for the requirement for local epidermal fatty acid synthesis in barrier homeostasis.

In animals with a normal barrier, TOFA treatment, even when applied for several days, does not affect barrier function. As described above, TOFA does not affect lipid synthesis in animals with a normal barrier, and therefore it is not surprising that this drug does not alter barrier function when applied to normal epidermis.

Prior studies and the experiments shown in Table IV (line 1 vs. line 3) demonstrate that inhibition of cholesterol synthesis causes a delay in the early stages of barrier recovery $(7,8)$. We next assessed the effects of simultaneous inhibition of fatty acid and cholesterol synthesis. As shown in Table IV, coapplications of TOFA and the HMG CoA reductase inhibitor, fluvastatin, result in an even more striking impairment in barrier

Table IV. Effect of Inibiting Cholesterol and Fatty Acid Synthesis on Barrier Recovery

\begin{tabular}{lccc}
\hline & $2 \mathrm{~h}$ & $4 \mathrm{~h}$ & $6 \mathrm{~h}$ \\
\hline & \multicolumn{3}{c}{ percent maximal TEWL } \\
1) Vehicle $(n=30)$ & $80.5 \pm 2.9$ & $64.4 \pm 2.9$ & $46.5 \pm 3.0$ \\
2) TOFA $(n=22)$ & $101.4 \pm 3.6$ & $85.2 \pm 3.5$ & $74.6 \pm 5.5$ \\
3) Fluvastatin $(n=13)$ & $122.2 \pm 6.4$ & $80.2 \pm 3.8$ & $65.2 \pm 3.8$ \\
4) $\mathrm{T}+\mathrm{F}(n=23)$ & $130 \pm 5.9$ & $112 \pm 5.7$ & $108 \pm 6.1$ \\
5) $\mathrm{T}+\mathrm{F}+\mathrm{PA}$ & & & \\
$\quad+$ cholesterol $(n=12)$ & $62.6 \pm 3.4$ & $46.2 \pm 3.3$ & $36.7 \pm 3.3$ \\
6) $\mathrm{T}+\mathrm{F}+\mathrm{PA}$ & & & \\
$\quad+$ epicholesterol & & & \\
$\quad(n=12)$ & $137 \pm 10$ & $86.7 \pm 6.8$ & $71.9 \pm 8.0$ \\
1 vs. 2 & $P<0.001$ & $P<0.001$ & $P<0.001$ \\
1 vs. 3 & $P<0.001$ & $P<0.01$ & $P<0.001$ \\
1 vs. 4 & $P<0.001$ & $P<0.001$ & $P<0.001$ \\
2 vs. 4 & $P<0.001$ & $P<0.001$ & $P<0.001$ \\
3 vs. 4 & $\mathrm{NS}$ & $P<0.001$ & $P<0.001$ \\
4 vs. 5 & $P<0.001$ & $P<0.001$ & $P<0.001$ \\
4 vs. 6 & $\mathrm{NS}$ & $P<0.01$ & $P<0.05$ \\
5 vs. 6 & $P<0.001$ & $P<0.001$ & $P<0.001$ \\
& & &
\end{tabular}

T, TOFA; F, fluvastatin; PA, palmitic acid. recovery than is achieved with either inhibitor alone (line 4 vs. lines 2 or 3). Moreover, exogenous fatty acids and cholesterol applied with the two inhibitors result in normalization of barrier recovery (line 4 vs. line 5 ). In contrast, simultaneous applications of TOFA plus palmitate and fluvastatin plus epicholesterol (3- $\alpha$-cholesterol), an inert free sterol that does not substitute for cholesterol in forming lamellar membranes (16), results in barrier recovery rates comparable to fluvastatin alone (line 3 vs. line 6). Together, these results demonstrate that the inhibition of barrier recovery induced by TOFA and fluvastatin can be attributed to the inhibition of fatty acid and cholesterol synthesis, and again is not simply due to nonspecific toxicity. Moreover, the observation that epicholesterol cannot substitute for cholesterol shows that the corrective effects of coapplied lipids is not due to bulk hydrophobic properties of the lipid, but instead to specific requirements for each lipid species.

Lipid histochemistry of TOFA-treated animals. We next determined whether TOFA treatment delays the reaccumulation of stainable neutral lipids in the stratum corneum (Fig. 1, $A-D)$. As seen in Fig. $1 A$, before acetone treatment the stratum corneum displays intense green-gold fluorescence. Immediately after acetone treatment this band is lost, and the stratum corneum is barely visible (Fig. $1 B$ ). Pilosebaceous structures in the dermis, however, continue to stain intensely, serving as a positive control (shown in Fig. $1 D$; see below). By $4 \mathrm{~h}$ after acetone treatment plus vehicle applications, greengold fluorescence is beginning to return to the stratum corneum (Fig. $1 C$ ). In contrast, TOFA treatment of acetone-perturbed skin resulted in a delay in the return of stainable neutral lipid to the stratum corneum (Fig. $1 D$ ); i.e., TOFA-treated samples appeared indistinguishable from samples obtained immediately after acetone treatment (Fig. $1 D$; compare Fig. $1 B$ ). These results demonstrate that the TOFA-induced inhibition of fatty acid synthesis results in a delay in the reappearance of stainable neutral lipid in the stratum corneum in parallel to the delay in barrier recovery.

Ultrastructural observations of TOFA-treated epidermis. To gain further insights into the mechanism by which TOFA inhibits barrier repair, we next examined the ultrastructure of TOFA-treated epidermis (Fig. 2, $A-E$ ). $2 \mathrm{~h}$ after vehicle treatment of acetone-disrupted skin, the appearance of lamellar bodies and their secreted contents at the stratum granulosum-stratum corneum interface appears comparable to normal (Fig. 2, $D$ and $E$ ). Moreover, normal lamellar bilayers are already present within the intercellular spaces in the lower to mid-stratum corneum (Fig. 2, D, double arrows). In contrast, TOFA treatment drastically alters the internal lamellar structure and organization of epidermal lamellar bodies (Fig. $2 C$ ). At the stratum granulosum-stratum corneum interface, the secreted contents of lamellar bodies appear disorganized and they only partially fill the intercellular spaces (Fig. $2 B$ ). Furthermore, the intercellular domains of the outer layers of TOFA-treated stratum corneum reveal a complete absence of normal bilayer structures; instead, fragments and whorls of lamellae partially fill the intercellular spaces, leaving a vacuolated appearance (Fig. $2 A$ ). However, when palmitate is coapplied with TOFA (Fig. $2 A,+P$ ), the internal structure of lamellar bodies is normalized (Fig. $2 A$, inserts below), and normal bilayers reappear within the stratum corneum interstices (Fig. $2 A$, insert above). These results show that inhibition of fatty acid synthesis by 

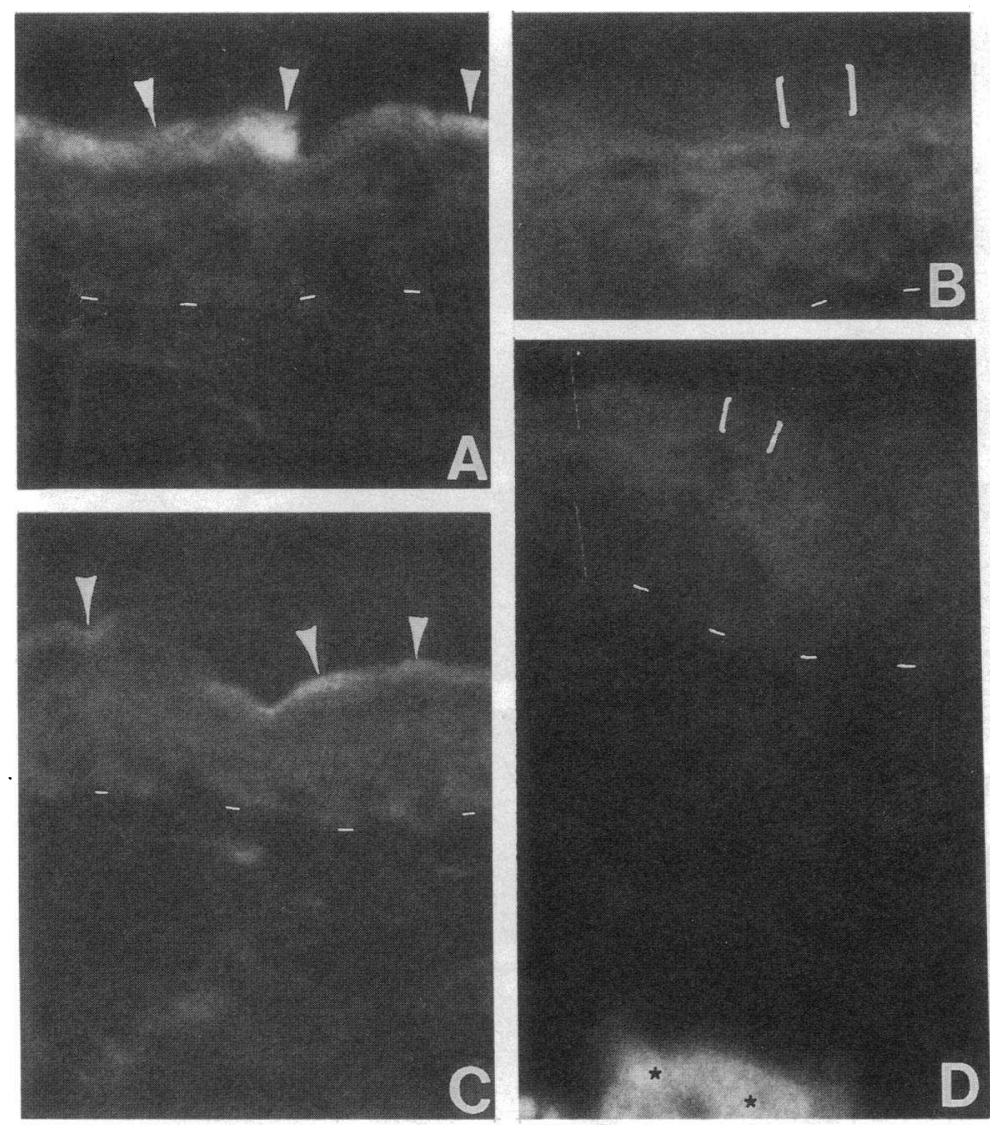

Figure 1. Frozen sections of hairless mice epidermis stained with the neutral lipid fluorophore, Nile red. $(A)$ Control, untreated skin: note intense green-gold fluorescence of stratum corneum (SC) (arrows). (B) Immediately after acetone treatment: note absence of SC staining (brackets). (C) $4 \mathrm{~h}$ after acetone treatment: note partial return of green-gold fluorescense in SC (arrows). (D) $4 \mathrm{~h}$ after acetone treatment plus one TOFA application: note lack of stainable lipid in SC (brackets), comparable to appearance immediately after acetone treatment. Also note positive staining of pilosebaceous structures in dermis (asterisks). Dashed line indicates dermal-epidermal junction. Magnification, 200.
TOFA leads to disruption of the lamellar body secretory system and the resultant intercellular bilayers in the stratum corneum.

\section{Discussion}

Previous studies have demonstrated that both cholesterol and sphingolipid synthesis in the epidermis are crucial for normal restoration of barrier function after acute disruption of the barrier (7-9). Inhibition of either epidermal cholesterol or sphingolipid synthesis results in the formation of abnormal lamellar bodies and a delay in the return of the respective lipid to the stratum corneum resulting in impaired barrier recovery (7-9). Most importantly, the inhibition of barrier repair can be overcome by topically providing the missing lipid, either cholesterol or sphingolipid, demonstrating that the inhibition of barrier recovery is not due to nonspecific toxicity, but rather the presence of both of these two lipid species is required for the formation of the water impermeable extracellular membranes of the stratum corneum (7-9).

The present study demonstrates that after acute disruption of the barrier, epidermal fatty acid synthesis is also crucial for normal restoration of barrier function. Topical treatment with TOFA inhibits epidermal fatty acid synthesis by $\sim 50 \%$ in both acetone-treated and tape-stripped animals; i.e., to rates less than or equal to levels in untreated epidermis. In contrast, topical TOFA treatment did not effect epidermal fatty acid synthesis in animals with an intact barrier. We suspect that the failure of TOFA treatment to inhibit epidermal fatty acid synthesis in animals with a normal barrier is due to the inability of TOFA to penetrate intact stratum corneum. Thus, disrupting the stratum corneum permeability barrier by either acetone treatment or tape stripping allows for the transcutaneous absorption of TOFA resulting in inhibition of fatty acid synthesis.

In addition to inhibiting epidermal fatty acid synthesis, topical TOFA treatment also causes a modest decrease in epidermal cholesterol synthesis, which did not achieve statistical significance in any of the experiments. This marked decrease in fatty acid synthesis with a modest decrease in cholesterol synthesis has also been noted in the liver after TOFA treatment (17-19).

A single topical application of TOFA significantly impairs barrier recovery after disruption by acetone treatment or tape stripping. That this delay can be attributed to deficiency of newly synthesized fatty acids is shown by the override experiments. Providing exogenous fatty acids by the topical coapplication of palmitate, simultaneously with TOFA, improves barrier recovery. In contrast, topical treatment with cholesterol has no significant beneficial effect on barrier recovery. Moreover, in other studies, we have shown that the application of palmitic acid alone after barrier disruption impairs rather than enhances barrier recovery (20). These observations strongly suggest that the delay in barrier recovery caused by TOFA is 

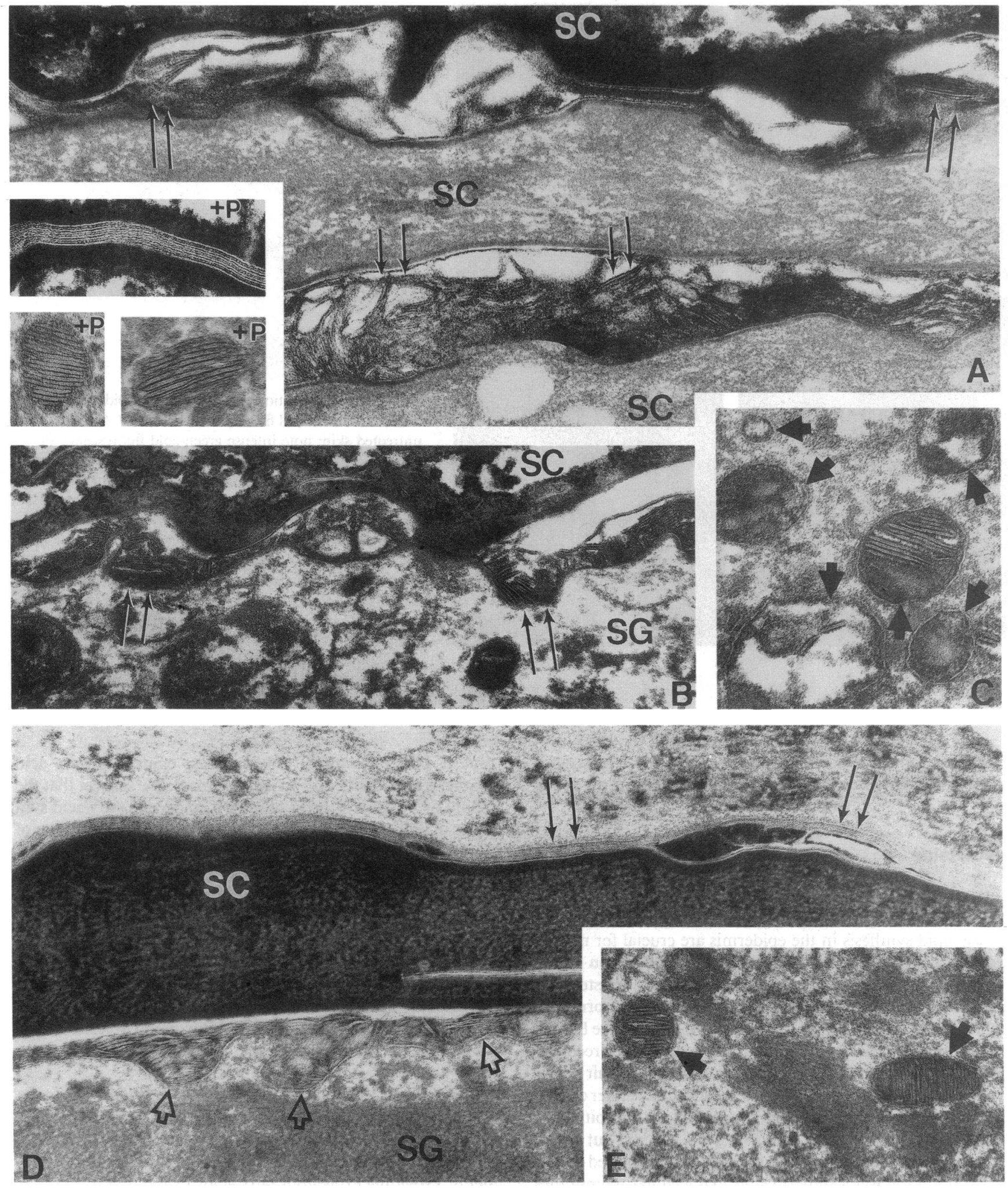

Figure 2. Ultrastructure of epidermis $2 \mathrm{~h}$ after TOFA ( \pm palmitate) treatment. $A-C$ show epidermis after TOFA treatment, while $D$ and $E$ show vehicle-treated controls. Note abnormal lamellar bodies with TOFA treatment $(C$, single arrows; compare normal lamellar bodies in $E)$. Secreted lamellar body contents at the stratum granulosum (SG)-strateum corneum (SC) interface only partially fill the intercellular domains (B, double arrows), leaving extensive clefts. At higher levels within the SC, the intercellular lamellar bilayers do not form normally; instead lamellar form short arrays or whorls $(A$, double arrows) leaving extensive clefts (compare normal SG-SC interface (open arrows) and lamellar bilayers (double arrows $)$ in $D$. Finally, coapplications of palmitate $(+P)$ with TOFA normalize the appearance of lamellar bodies $(A,+P$, lower insets $)$, as well as lamellar bilayer structure $(A,+P$, upper inset $) . A$ and insets, $\times 145,000 ; B, \times 102,500 ; C, \times 155,000 ; D, \times 86,000 ; E, \times 108,000$. 
specifically due to the inhibition of fatty acid synthesis and not due to nonspecific drug toxicity.

This and prior studies have demonstrated that, as with inhibition of fatty acid synthesis, the inhibition of cholesterol synthesis also impairs the early phases of barrier recovery $(7,8)$. These studies further show that when TOFA and fluvastatin, an inhibitor of cholesterol synthesis, are coapplied there is a marked inhibition of barrier recovery. In fact, with the two inhibitors together there is no recovery of barrier function during the $6 \mathrm{~h}$ of study. Notably, providing palmitate and cholesterol simultaneously with the two inhibitors restores barrier recovery to normal. In contrast, providing palmitate and epicholesterol, an inert free sterol that can not substitute for cholesterol in forming membrane structures (16), results in barrier recovery rates comparable to fluvastatin treatment alone. These observations provide still further evidence that the delay in barrier recovery is specifically linked to inhibition of epidermal lipid synthesis.

Fatty acids are substrates for the formation of sphingolipids, and as stated above, sphingolipids are important constituents of the stratum corneum permeability barrier. However, it is unlikely that the effect of TOFA on barrier recovery observed here can be attributed to inhibition of sphingolipid formation because prior studies have suggested that preformed stores of sphingolipids appear to suffice for the early stages of barrier recovery $(5,9)$. Sphingolipid synthesis is only stimulated at later time points after barrier disruption (5), and a specific inhibitor of sphingolipid synthesis, $\beta$-chloroalanine, produced a defect in barrier recovery only during the later phases of barrier repair (12-24 h) (9). In contrast, inhibiting epidermal fatty acid synthesis with TOFA inhibits the early phases of barrier recovery.

As reported in previous publications, topical acetone treatment extracts lipids from the stratum corneum resulting in the disappearance of stainable neutral lipids (Fig. $1, B$ vs. $A)(7$, $14)$. Within a few hours after acetone treatment, stainable neutral lipids begin to return to the stratum corneum (Fig. 1 C) (14), in parallel to the restoration of barrier function. In contrast, in TOFA-treated animals, there is a delay in the return of neutral lipids to the stratum corneum (Fig. $1, D$ vs. $C$ ). Thus, inhibiting fatty acid synthesis delays the return of lipids to the stratum corneum which may account for the impaired barrier recovery.

The basis for this delay in the return of lipids to the stratum corneum in TOFA-treated animals was revealed by the ultrastructural studies. As discussed in detail in prior publications, the exocytosis of lamellar bodies is an important route by which lipids are delivered to the extracellular domains of the stratum corneum (reviewed in 3, 21-23). The lamellar body secretory system appears to be activated by barrier disruption because lamellar material appears in greater than normal quantities at the stratum granulosum-stratum corneum interface (3). In TOFA-treated animals, both the internal structure and organization of lamellar bodies are disrupted (Fig. $2 C$ ). Furthermore, the quantities of secreted lamellar body material at the stratum granulosum-stratum corneum interface are diminished, and the organization of this material is also abnormal within the intercellular spaces at all levels of the stratum corneum (Fig. 2, $A$ and $B$ ). However, when palmitate is coapplied with TOFA, the internal structure of lamellar bodies is normalized (Fig. $2 A$, inset), and normal bilayers appear at all levels within the stratum corneum interstices by $4 \mathrm{~h}$. These observations suggest that the TOFA-induced deficiency in nascent fatty acids leads to alterations in lamellar body content. These abnormalities in the lamellar body secretory system may account for the delay in both the return of stainable lipids to the stratum corneum and the delayed restoration of barrier function with TOFA treatment. Remarkably, all of these changes in lamellar body internal structure and stratum corneum intercellular lamellar organization occur and are reversed within $4 \mathrm{~h}$, once again emphasizing both the rapidity of the repair response and the fluidity of the intercellular domains. In recent studies, we have shown that substantial percutaneous transport, delivery to keratinocytes, and processing through the lamellar body secretory system occurs by $2 \mathrm{~h}$ after topical lipid application to acetone-treated murine skin (20).

In summary, these results demonstrate first a requirement for bulk fatty acids for permeability barrier homeostasis, and second, the central role of the lamellar body secretory system in mediating changes in barrier function. Third and finally, these results demonstrate that diminution of any of the three key lipids that form the extracellular, lipid-enriched membranes of the stratum corneum results in a delay in the return of lipids to the stratum corneum and an impairment in the recovery of barrier function.

\section{Acknowledgments}

We thank P. Herranz for excellent secretarial assistance.

This work was supported by grants from the Research Service of the Department of Veterans Affairs and the National Institutes of Health (AR-19098 and AR-39639).

\section{References}

1. Elias, P. M. 1983. Epidermal lipids, barrier function and desquamation. $J$ Invest. Dermatol. 80:44-49.

2. Feingold, K. R. 1991. The regulation and role of epidermal lipid synthesis. Adv. Lipid Res. 24:57-82.

3. Menon, G. K., K. R. Feingold, and P. M. Elias. 1992. The lamellar body secretory response to barrier disruption. J. Invest. Dermatol. 98:279-289.

4. Menon, G. K., K. R. Feingold, A. H. Moser, B. E. Brown, and P. M. Elias. 1985. De novo sterologenesis in the skin. Il. Regulation by cutaneous barrier requirements. J. Lipid Res. 26:418-427.

5. Grubauer, G., K. R. Feingold, and P. M. Elias. 1987. The relationship of epidermal lipogenesis to cutaneous barrier function. J. Lipid Res. 28:746-752.

6. Holleran, W. M., K. R. Feingold, M. Mao-Qiang, W. N. Gao, J. M. Lee, and P. M. Elias. 1991. Regulation of epidermal sphingolipid synthesis by permeability barrier function. J. Lipid Res. 32:1151-1158.

7. Feingold, K. R., M. Mao-Qiang, W. N. Gao, G. K. Menon, and P. M. Elias. 1991. Cholesterol synthesis is required for cutaneous barrier function in mice. $J$. Clin. Invest. 86:1738-1745.

8. Menon, G. K., K. R. Feingold, M. Mao-Qiang, M. Schaude, and P. M. Elias. 1992. Structural basis for the barrier abnormality following inhibition of HMG CoA reductase in murine epidermis. J. Invest. Dermatol. 98:209-219.

9. Holleran, W. M., M. Mao-Qiang, W. N. Gao, G. K. Menon, P. M. Elias, and K. R. Feingold. 1991. Sphingolipids are required for mammalian barrier function: inhibition of sphingolipid synthesis delays barrier recovery after acute perturbation. J. Clin. Invest. 88:1338-1345.

10. Prottey, C. 1976. Essential fatty acids and the skin. Br. J. Dermatol. 94:579-587

11. Wertz, P. W., and D. T. Downing. 1982. Glycolipids in mammalian epidermis: structure and function in the water barrier. Science (Wash. DC). 217:1261-1262.

12. Monger, D. J., M. L. Williams, K. R. Feingold, B. E. Brown, and P. M. Elias. 1988. Localization of sites of lipid biosynthesis in mammalian epidermis. $J$. Lipid Res. 29:603-612. 
13. Proksch, E., P. M. Elias, and K. R. Feingold. 1990. Regulation of 3-hydroxy-3-methylglutaryl-coenzyme $A$ reductase activity in murine epidermis: modulation of enzyme content and activation state by barrier requirements. $J$. Clin. Invest. 85:874-882.

14. Grubauer, G., P. M. Elias, and K. R. Feingold. 1989. Transepidermal water loss: the signal for recovery of barrier structure and function. J. Lipid Res. 30:579-585.

15. Hou, S. Y. E., A. K. Mitra, S. H. White, G. K. Menon, R. Ghadially, and P. M. Elias. 1991. Membrane structures in normal and essential fatty acid deficient stratum corneum: characterization by ruthenium tetroxide staining and x-ray diffraction. J. Invest. Dermatol. 96:215-223.

16. Elias, P. M., D. S. Friend, and J. Goerke. 1979. Membrane sterol heterogeneity: freeze fracture detection with saponins and filipin. J. Histochem. Cytochem. 27:1247-1260.

17. Panek, E., G. A. Cook, and N. W. Cornell. 1977. Inhibition by 5-(tetradexyloxy)-2-furoic acid of fatty acid and cholesterol synthesis in isolated rat hepatocytes. Lipids. 12:814-818.
18. McCune, S. A., and R. A. Harris. 1979. Mechanism responsible for 5-(tetradexyloxy)-2-furoic acid inhibition of hepatic lipogenesis. J. Biol. Chem. 254:10095-10101.

19. Fukuda, N., and J. A. Ontko. 1984. Interactions between fatty acid synthesis, oxidation, and esterification in the production of triglyceride rich lipoproteins by the liver. J. Lipid Res. 25:831-842.

20. Mao-Qiang, M., K. R. Feingold, and P. M. Elias. 1993. Effects of exogenous lipids on permeability barrier recovery in acetone treated murine skin. Arch. Dermatol. In press.

21. Odland, G. P., and K. Holbrook. 1987. The lamellar granules of epidermis. Curr. Probl. Dermatol. 9:29-41.

22. Landmann, L. 1988. The epidermal permeability barrier. Anat. Embryol. 178:1-13.

23. Elias, P. M., and G. K. Menon. 1991. Structural and lipid biochemical correlates of the epidermal permeability barrier. Adv. Lipid Res. 24:1-26. 